\title{
El sistema iberoamericano y los Derechos Humanos: una atmósfera favorable en el quehacer de la Corte Interamericana de Derechos Humanos
}

\section{The Ibero-American System and Human Rights: a Favourable Atmosphere in the Work of the Inter-American Court of Human Rights}

\author{
Elena C. Díaz Galán ${ }^{1}$ \\ Universidad Rey Juan Carlos de Madrid (España) \\ ORCID: https://orcid.org/0000-0003-0914-8944
}

Recibido: 18-01-2020

Aceptado: 24-11-2020

\section{Resumen}

La Conferencia iberoamericana constituye un espacio de diálogo, concertación y cooperación que asume como principio esencial la vigencia de los derechos humanos. Estos derechos conforman un ámbito de actuación del sistema iberoamericano. Los instrumentos aprobados por la Conferencia y otras instancias iberoamericanas proporcionan indicaciones políticas y jurídicas en el campo de los derechos humanos que deben ser desarrolladas a través de

\footnotetext{
${ }^{1}$ (elena.galan@urjc.es). Profesora Ayudante Doctor (acreditada Contratado Doctor) Derecho Internacional Público y Relaciones Internacionales. Universidad Rey Juan Carlos de Madrid. Coordinadora del Grado en Relaciones Internacionales. Este trabajo se ha elaborado en el marco del Grupo de Investigación de Alto Rendimiento en Libertad, Seguridad y Ciudadanía en el Orden Internacional (INTERCIVITAS), de la Universidad Rey Juan Carlos.

Entre las líneas de investigación: Derechos Humanos, Organizaciones internacionales, Asilo diplomático, cooperación e integración en Latinoamérica, seguridad internacional y desarrollo sostenible.

Entre las publicaciones destacan: La conformación jurídica de las organizaciones internacionales. De las conferencias internacionales a las uniones administrativas, Editorial Aranzadi- Thomson Reuter, Pamplona, 2018; "Tratado sobre la prohibición de las armas nucleares (TPAN): Un paso más en la ilicitud del empleo del arma nuclear", Revista de Estudios en Seguridad Internacional (RESI), vol. 5, n'. 2, 2019, pp. 39-58; y "La indefinición jurídica del asilo diplomático al hilo de la práctica internacional: -Una figura olvidada-”, Anuario Español de Derecho Internacional (AEDI), nº. 35, 2019, pp. 405-450.
} 
iniciativas, acciones, programas y agendas de cooperación. Los derechos de contenido social merecen una especial atención en las Cumbres iberoamericanas. Todo esto debería tener una influencia en las posiciones de la Corte Interamericana de Derechos Humanos, particularmente cuando aborde asuntos en los que queden afectados derechos económicos, sociales y culturales, tal como viene haciendo al hilo de recientes sentencias en la materia. La labor del sistema iberoamericano en los campos relativos a las migraciones y la cultura será especialmente interesante en el quehacer de la Corte IDH para determinar el contenido y alcance de los derechos que se reconocen y protegen en el sistema interamericano.

Palabras-clave: Conferencia Iberoamericana, Derechos humanos, Derechos sociales, Corte Interamericana de Derechos humanos.

\section{Abstract}

The Ibero-American Conference is a forum for dialogue, consultation and cooperation in which the observance of human rights is an essential principle. These rights are an area of action of the Ibero-American system. The instruments adopted by the Conference and other Ibero-American instances provide political and legal indications in the field of human rights that must be developed through initiatives, actions, programmes and agendas of cooperation. Social rights deserve special attention at the Ibero-American Summits. All this should have an influence on the positions of the Inter-American Court of Human Rights, particularly when it deals with cases in which are affected economic, social and cultural rights, as it has been done in the wake of recent judgments on the matter. The work of the Ibero-American system in the fields of migration and culture will be of particular interest to the Inter-American Court in determining the content and scope of the rights recognized and protected in the inter-American system.

Key-words: Ibero-American Conference, Human Rights, Social Rights, Inter-American Court of Human Rights.

\section{Introducción}

Por primera vez, los Jefes de Estado y de Gobierno de los países iberoamericanos se reúnen en una Conferencia Internacional, en 1991, con el propósito de promover una instancia de diálogo, concertación política y cooperación internacional. Los objetivos quedarán plasmados en la Declaración de Guadalajara y se irán añadiendo o perfilando nuevas finalidades en las sucesivas Cumbres hasta la última hasta ahora, que ha tenido lugar en La 
Antigua (Guatemala), en 2018. Las Cumbres han aprobado Declaraciones, Planes y Programas de Acción, Comunicados y otros instrumentos de diversa naturaleza, contenido y alcance y, además, representan la cúspide de todo un sistema integrado por distintas instancias, Organizaciones internacionales y conferencias de todo tipo ${ }^{2}$. De ahí nace el "acervo iberoamericano".

Para el entonces Secretario General Iberoamericano, Enrique V. Iglesias: A lo largo de sus años de vida "las Cumbres Iberoamericanas han ido consagrando (...) una serie de valores, principios y objetivos, que son la razón de ser de la existencia de la Comunidad Iberoamericana y que deben ser fuente inspiradora de las políticas de nuestros países, tanto a nivel interno como internacional.

Siendo así que "esto es lo que las propias Cumbres han consagrado como acervo iberoamericano, que constituye un auténtico código de conducta de los Estados (...)",3.

Los mandatos de las Cumbres y los postulados de las instancias del sistema iberoamericano van más allá, por ende, de cualquier planteamiento declarativo y permiten penetrar en sectores tangibles de la cooperación iberoamericana.

El acervo iberoamericano está integrado por las raíces históricas y culturales que comparten los Estados y pueblos que integran la Comunidad Iberoamericana de Naciones (CIN) pero, también, por valores, principios, guías de conducta, comportamientos y políticas que derivan del quehacer común y que acopian los postulados políticos y normativos que expresan los Estados en las Cumbres y en el resto de las instancias iberoamericanas. Para C. del Arenal

el acervo iberoamericano está constituido (...) por un conjunto de principios, valores y compromisos que encuentran sus bases no solo en una historia, una comunidad y una cultura común, labrada a lo largo del tiempo, sino también en la propia dinámica de diálogo, concertación y cooperación iberoamericana puesta en marcha por las Cumbres Iberoamericanas ${ }^{4}$.

\footnotetext{
${ }^{2}$ En el Informe presentado a la SEGIB por los Profesores Fernando Mariño Menéndez y Cástor Miguel Díaz Barrado, de 2006, titulado "Informe sobre las formas de incorporación y participación en el sistema iberoamericano representado por la Conferencia Iberoamericana. (Criterios, Modalidades, Estatutos y Procedimiento de participación)", se dice que "en una aproximación genérica pueden considerarse como elementos integrantes del -Sistema Iberoamericano-, ya sean públicos o privados, internacionales o internos, los Estados de raíz iberoamericana, sus pueblos iberoamericanos y sus ciudadanos, las Organizaciones Internacionales iberoamericanas, las organizaciones no gubernamentales surgidas o integradas dentro del espacio iberoamericano y las demás instituciones públicas y privadas que actúen dentro del mismo". Agradezco a los autores haberlo facilitado para su consulta.

${ }^{3}$ Celestino del Arenal, El acervo iberoamericano, Valores, principios y objetivos de la comunidad iberoamericana, Secretaria General Iberoamericana, Madrid, 2006, p. 7. Es conveniente la lectura también de: Yago Pico de Coaña y de Valicourt, "El valor de los principios en la comunidad iberoamericana", Cuadernos de Estrategia, n. 126, 2004, pp. 149-203.

${ }^{4}$ Celestino del Arenal, El acervo iberoamericano, op. cit., p. 14.
} 
Pues bien, dentro de las realizaciones de las Cumbres y de todo el sistema iberoamericano hay que prestar una especial atención al sector relativo a los derechos humanos. En particular, este sector está presente en los instrumentos de las Cumbres y será una constante preocupación en muchas de las dimensiones de la cooperación iberoamericana, lo que permite reflexionar sobre su eventual influencia en los sistemas regionales de protección de los derechos humanos en los que participan los Estados iberoamericanos y, en concreto, en la labor que realiza la Corte Interamericana de Derechos Humanos (Corte IDH)

La notable escasez de trabajos doctrinales sobre la realidad iberoamericana en términos institucionales se acrecienta cuando se trata del aspecto específico sobre los derechos humanos ${ }^{5}$. En especial, las repetidas alusiones en los instrumentos adoptados por las Cumbres a cuestiones de derechos humanos no han venido acompañadas de un atento estudio por la doctrina científica, lo que ha provocado que no se hayan extraído, a efectos de interpretación, todas las consecuencias que se han querido otorgar a este principio y a este ámbito de la cooperación iberoamericana. En cualquier caso, se deben reconocer los contenidos de los compromisos político-jurídicos en materia de derechos humanos de los Estados de la región iberoamericana e intentar trasladarlos a otras instancias de reconocimiento y protección de estos derechos. En este sentido, queda pendiente subrayar la vinculación entre el sistema iberoamericano y el sistema interamericano en materia de derechos humanos.

Este campo de actuación debe ser contemplado al menos desde una triple dimensión: por una parte, la calificación que merecen los derechos humanos como valor, principio o guía de conducta de los Estados en sus relaciones mutuas y en la proyección internacional de la CIN, lo que hace pensar que esto repercutirá en los órganos de aplicación de los sistemas regionales de protección de los derechos humanos en los que participan los Estados de la región. Por otra parte, puesto que los derechos humanos son un sector prioritario de la cooperación iberoamericana que recibe respuestas políticas y normativas, será posible extraer consecuencias en algunos ámbitos que son primordiales para los países de la región, como la cultura, la educación, la seguridad social o las migraciones y, en general, en todo lo relativo a los derechos de contenido económico, social y cultural. Esto permitirá que los órganos regionales de protección de los derechos humanos en los que participan los Estados iberoamericanos puedan sustentar sus posiciones en instrumentos del sistema iberoamericano. Por último, la posición geopolítica que ocupa

\footnotetext{
${ }^{5}$ Quizá solo dos trabajos se detienen más específicamente en este campo: Carlos Fernández Liesa, "La democracia y los derechos humanos en el espacio eurolatinoamericano e iberoamericano", en Perspectivas sobre las relaciones entre la Unión Europea y América Latina, Universidad Carlos III/ BOE, Madrid, 2008, pp. 51-77; y Celestino del Arenal, "Democracia y derechos humanos en las cumbres iberoamericanas. Teoría y práctica”, en La Cumbre de Cádiz y las relaciones de España con América Latina, Madrid, Colección Escuela Diplomática, 19, 2013, pp. 147-163.
} 
la comunidad iberoamericana y el entorno jurídico que rodea su actividad hacen difícil profundizar en el establecimiento de una normativa propia sobre derechos humanos y en la instauración de mecanismos institucionales que dieran eficacia a las políticas en esta materia, sin embargo conviene determinar si de manera explícita o implícita los resultados de las Cumbres y del sistema iberoamericano en materia de derechos humanos inciden en el quehacer de los órganos de protección de estos derechos y, en concreto, en la tarea que realiza la Corte IDH.

\section{La necesidad de un marco de cooperación entre el sistema iberoamericano y la Corte Interamericana de derechos humanos}

La vigencia de los derechos humanos es un pilar fundamental de la Conferencia iberoamericana y, por ende, de la comunidad a la que representa y del sistema iberoamericano. Por esto se debe dar efectividad a los compromisos de los Estados iberoamericanos en esta materia. Sería inútil acoger el respeto de los derechos humanos como sustento esencial de estas instancias sin que este reconocimiento tuviera algún tipo de trascendencia política y jurídica que se podría plasmar en la elaboración de políticas, guías de conductas o compromisos políticos entre los Estados pero también en las posiciones de los órganos regionales encargados de la protección de los derechos humanos en los que participan los Estados de la región. Esto es precisamente lo que ha sucedido, aunque de modo parcial. Así, las declaraciones finales de las Cumbres y los Planes de Acción se detienen en la indicación de programas, medidas, orientaciones y propuestas de actuación que guardan relación con el ámbito de los derechos humanos. Pero, sobre todo, la vinculación con los derechos humanos se advierte en los escasos acuerdos político-jurídicos que ha adoptado la Conferencia iberoamericana, que ha quedado plasmada en instrumentos independientes y autónomos sobre algunas materias específicas de la cooperación iberoamericana. La finalidad sería que todas estas acciones se hicieran efectivas bien a través de los ordenamientos jurídicos internos de los Estados de la región y mediante el quehacer de los órganos jurisdiccionales de los sistemas regionales establecidos, principalmente en el espacio americano.

Los dos objetivos se podrían comprobar en el análisis de los resultados de las Cumbres. Por una parte, la obligación de fortalecer los mecanismos nacionales de defensa de los derechos humanos se recoge en la Declaración de Guadalajara, cuando ampara el compromiso de "crear, y en su caso consolidar, en nuestros respectivos países, mecanismos nacionales de promoción, protección y defensa de los derechos humanos, así como establecer una 
cercana colaboración entre los mismos" $" \mathrm{y}$, de nuevo, en el Documento de Conclusiones de la Cumbre de Madrid, de 1992, al felicitarse por la creación “de mecanismos nacionales de protección de los derechos humanos en varios países miembros" ". Es decir, traducir en los ordenamientos jurídicos internos la visión iberoamericana en materia de derechos humanos e instaurar mecanismos singulares que robustezcan los procedimientos de protección de estos derechos. Por otra parte, prestar la debida atención a la cooperación internacional para reforzar la defensa de los derechos humanos como lo anunció la Declaración de Guadalajara que aboga por

fortalecer la cooperación entre gobiernos y entidades de la sociedad civil y organismos multilaterales competentes en materia de derechos humanos, y fomentar la plena adhesión a los instrumentos internacionales de promoción y de protección de estos derechos tanto de carácter universal como regional ${ }^{8}$.

Lo que no excluye los tribunales regionales encargados de garantizar los derechos humanos. Aquí se trataría de incorporar el acervo iberoamericano en los logros de los sistemas de reconocimiento y protección en los que participan los Estados de la región.

En particular, los Estados iberoamericanos tienen a su disposición los mecanismos de garantía de los derechos humanos más evolucionados y articulados del planeta. En especial, el Tribunal Europeo de Derechos Humanos (TEDH) para los Estados de Europa y la Corte IDH (además de la Comisión Interamericana) para los Estados de América. La región iberoamericana, con identidad y características propias, se ve entreverada en este caso por la pertenencia de los Estados a la región europea y americana. Desde ahí, la Conferencia iberoamericana podría animar e impulsar la plena participación de los Estados de la región en estos sistemas y vigorizar la protección de los derechos humanos en Iberoamérica. Por ello, las Conclusiones de Madrid, de 1992, evocan la importancia del "desarrollo del sistema regional de protección de los derechos humanos y la efectividad de su aplicación" y dejan constancia de que la "cooperación entre el sistema europeo de protección de los derechos humanos y el interamericano" (...) "ha sido ejemplar y debe continuar desenvolviéndose",

En el caso particular, la Corte IDH se encuentra en una situación ideal para acoger resultados de la Conferencia Iberoamericana en el sector de los derechos humanos, al menos, por dos razones fundamentales: primera, porque todos los Estados iberoamericanos de raíz americana, excepto $\mathrm{Cuba}^{10}$, son partes en la

\footnotetext{
${ }^{6}$ SEGIB: https://www.segib.org/?document=declaracion-de-guadalajara.

${ }^{7}$ SEGIB: https://www.segib.org/?document=declaracion-de-madrid.

${ }^{8}$ SEGIB: https://www.segib.org/?document=declaracion-de-guadalajara.

${ }^{9}$ SEGIB: https://www.segib.org/?document=declaracion-de-madrid.

10 Respecto al caso particular de Venezuela véase lo que se indica en el estado de firmas y
} 
Convención americana sobre derechos humanos, de 1969, y asimismo han aceptado la competencia de la Corte. Esto hace que los postulados que estos Estados sostengan en otras instancias internacionales y, sobre todo, en aquellas en las que ellos constituyan la mayoría, como es el caso de la Conferencia iberoamericana, pueden ser recogidos sin dificultad por la Corte interamericana. Segunda, que la Corte IDH ha abierto un espacio más amplio que el TEDH para el reconocimiento y garantía de los derechos de contenido económico, social y cultural, siendo así que la Conferencia iberoamericana sitúa el énfasis en los derechos de este tipo y aborda ámbitos íntimamente relacionados con el ejercicio de estos derechos.

En cualquier caso, la posición de la Conferencia iberoamericana ha sido clara en materia de derechos humanos y, por ello, la Corte IDH no debe tener duda alguna del significado que los Estados de la región han querido dar al reconocimiento de estos derechos $\mathrm{y}$, desde ahí, argumentar sus decisiones también con base en los pronunciamientos del sistema iberoamericano. Desde luego, el instrumento constitutivo de la Conferencia Iberoamericana sitúa en la cúspide de los valores y principios el respeto de los derechos humanos. La Declaración de Guadalajara asevera con convicción que la "comunidad se asienta en la democracia, el respeto a los derechos humanos y en las libertades fundamentales". Para afirmar, con posterioridad, el compromiso "con el desarrollo económico y social de nuestros pueblos, la plena vigencia de los derechos humanos, la ampliación de los cauces democráticos, el fortalecimiento de nuestros sistemas institucionales y el respeto de las normas de derecho internacional" 11 .

El discurso del instrumento constitutivo en este campo puede ser acogido por la Corte IDH. Ello se explicaría, al menos, por dos motivos de carácter estructural: en primer lugar, el momento histórico en el que se adopta la Declaración de Guadalajara coincide con un periodo de la comunidad internacional en el que se asegura la existencia de determinados valores como la democracia, el desarrollo económico y social y la protección de los derechos humanos. La inclusión de los derechos humanos en la Primera Cumbre iberoamericana es una expresión más del sentir de la comunidad internacional en ese periodo pero, en este caso, encuentra reflejo singular en el espacio iberoamericano, siendo así que prácticamente todos los Estados que

ratificaciones de la Convención Americana de Derechos Humanos. En concreto, se dice que "el 31 de julio de 2019, la República Bolivariana de Venezuela depositó el instrumento de ratificación de la Convención Americana sobre Derechos Humanos, en la sede de la OEA, en Washington, D. C., Estados Unidos". Este instrumento de ratificación reconoce "de manera incondicional como obligatoria de pleno derecho y sin convención especial la competencia y el poder jurisdiccional de la Corte Interamericana de Derechos Humanos (...), OEA:

https://www.oas.org/dil/esp/tratados_B32_Convencion_Americana_sobre_Derechos_Humanos firmas.htm

${ }^{11}$ SEGIB: https://www.segib.org/?document=declaracion-de-guadalajara.

Araucaria. Revista Iberoamericana de Filosofia, Política, Humanidades y Relaciones Internacionales, año $23, \mathrm{n}^{\circ} 46$. Primer cuatrimestre de 2021. Pp. 631-652. ISSN 1575-6823 e-ISSN 2340-2199 https://dx.doi.org/10.12795/araucaria.2021.i46.31 
participan en la Cumbre Iberoamericana, de 1991, aceptan las normas de la Convención Americana de derechos humanos. En segundo lugar, a veces no se le otorga la importancia debida a los elementos constitutivos que trazan la creación de la CIN tal y como se concibe a partir de 1991. La existencia de una comunidad histórica y cultural se enriquece con componentes de tipo político y con argumentos de naturaleza jurídica ${ }^{12}$. La Conferencia iberoamericana sobrepasa el discurso tradicional e incorpora como aspectos decisivos para justificar las reuniones entre los países iberoamericanos la democracia, el respeto de los derechos humanos y la vigencia del Derecho Internacional, a los que se debe añadir el desarrollo económico y social. Todo ello, a pesar de que la Declaración de 1991 no le dedique un apartado específico a la vigencia de los derechos humanos. La Conferencia refuerza así la posición que tienen los Estados en los respectivos sistemas regionales de protección de los derechos humanos $\mathrm{y}$, en especial, en el sistema interamericano.

El contenido de las referencias a los derechos humanos en la Declaración de Guadalajara tiene tal intensidad que no es preciso acudir a interpretaciones complicadas. Las dos Cumbres posteriores celebradas en Madrid, de 1992, y en Salvador de Bahía, de 1993 reiteran, aunque también precisan, el significado que se le otorga a la defensa de los derechos humanos en esta expresión de la realidad iberoamericana. El Documento de Conclusiones de Madrid recuerda el compromiso de los Estados iberoamericanos "con la democracia representativa, el respeto a los derechos humanos y las libertades fundamentales como pilares que son de nuestra comunidad" ". En la misma línea se pronuncia el Documento Final de Salvador de Bahía al expresar el "pleno compromiso con la democracia representativa, el respeto, la defensa y la promoción de los Derechos Humanos y de las libertades fundamentales", recalcando que "son objetivos básicos de la comunidad de naciones aquí reunidas y factores integrantes de cualquier política de cooperación"14.

La vigencia de los derechos humanos estará siempre presente como objetivo de la comunidad iberoamericana y como principio esencial de las relaciones entre los países iberoamericanos en cualquiera de los ámbitos en que se produzcan, también en el seno de los sistemas regionales de garantía

${ }^{12}$ Ernesto José Rey Caro, "Vigencia del Derecho Internacional en la Comunidad Iberoamericana de Naciones", Foro de reflexión sobre el espacio jurídico iberoamericano: realizaciones y propuestas, Madrid, Universidad Rey Juan Carlos 2004, pp. 19-36.

${ }^{13}$ Hasta tal punto que se dice que esta Declaración tiene "el objetivo de refrendar el compromiso con los principios y objetivos enunciados en su reunión fundacional", SEGIB: https://www.segib. org/?document=declaracion-de-madrid.

${ }^{14}$ SEGIB: https://www.segib.org/? document=declaracion-de-salvador-de-bahia. Una de las redacciones más acabadas es la que recoge la Declaración de Lima de 2001: "Los valores y principios compartidos que nos identifican conforman el acervo constitutivo de nuestra comunidad, y son coincidentes con los principios universales de la Carta de las Naciones Unidas, en especial, (...) el respeto y la promoción de los derechos humanos (...)", SEGIB: https://www.segib. org/?document=declaracion-de-lima.

Araucaria. Revista Iberoamericana de Filosofia, Politica, Humanidades y Relaciones Internacionales, año $23, \mathrm{n}^{\circ} 46$. Primer cuatrimestre de 2021. Pp. 631-652. ISSN 1575-6823 e-ISSN 2340-2199 https://dx.doi.org/10.12795/araucaria.2021.i46.31 
de estos derechos. Los términos empleados por la Conferencia iberoamericana sobrepasan el terreno de la retórica y resultan imprescindibles para reconocer y detallar los valores y principios en los que se asienta el quehacer de esta Conferencia. La doctrina más reputada así lo ha reconocido. Para C. del Arenal y A. Nájera "la Comunidad Iberoamericana de Naciones tiene, así, como proyecto, unos valores y objetivos, que son la libertad, el respeto de los derechos humanos, la paz, el desarrollo, la cooperación y la solidaridad"15; y C. M. Díaz Barrado sostiene que los Estados iberoamericanos "han prodigado expresiones de reafirmación y apoyo a los derechos humanos básicos, de tal modo que la defensa de estos derechos se configura como un principio fundamental de la Comunidad (...)"'16.

La rotundidad de estos pronunciamientos conduce, sin embargo, a reflexionar en el terreno de la efectividad para que se alcancen logros y resultados que se produzcan en esta materia en el sistema iberoamericano. La dificultad para que la CIN disponga de un órgano jurisdiccional encargado de garantizar el respeto de los derechos que se reconocen en el espacio iberoamericano se debe, en gran medida, a que los Estados de la región ya disponen de órganos de este tipo en los sistemas regionales europeo y americano de protección de los derechos humanos. En cualquier caso, habría que superar la situación descrita por C. del Arenal, para quien

\begin{abstract}
si el acervo consagrado por las cumbres iberoamericanas consagra formalmente la democracia y los derechos humanos (...), no sucede lo mismo cuando se desciende a la aplicación práctica de esos valores en relación a la realidad de los países iberoamericanos. Es en este ámbito práctico en el que surgen una serie de interrogantes en cuanto a la efectiva vigencia y defensa de la democracia y los derechos humanos por parte de las cumbres ${ }^{17}$.
\end{abstract}

Para lograr la efectividad se abren, al menos, dos vías:

La primera sería establecer cauces permanentes de colaboración iberoamericana entre los dos sistemas regionales de protección de los derechos humanos, en lo que se ha avanzado muy poco a pesar de que la Conferencia iberoamericana ocupa un lugar único y privilegiado para aclarar y robustecer las relaciones entre los órganos de garantía de ambos sistemas. En este sentido, Enrique V. Iglesias afirmó que "los sistemas regionales de protección de los derechos humanos constituyen un elemento esencial en la promoción y vigencia de los derechos humanos en Iberoamérica"18,

\footnotetext{
${ }^{15}$ Celestino del Arenal y Alfonso Nájera, La Comunidad Iberoamericana de Naciones: (pasado, presente y futuro de la política iberoamericana de España), Madrid, CEDEAL, 1990, pp. 33 ss.

${ }^{16}$ Cástor Miguel Díaz Barrado, Perfiles de la Comunidad Iberoamericana de Naciones (A la luz de las Cumbres Iberoamericanas, Tribuna Americana, Casa de América, Cáceres, 1994, p. 107.

${ }^{17}$ Celestino del Arenal, Democracia y derechos humanos Op. Cit., p. 155.

${ }^{18}$ Foro "Los desafíos actuales de la protección Internacional de los Derechos Humanos desde una
} 
pero se precisan algunos pasos más decisivos para convertir ese ideal en una verdadera cooperación entre sistemas que incorpore las señas de identidad de la realidad iberoamericana. La voluntad y el fundamento de esa eventual cooperación se puede sustentar en los postulados de la Conferencia iberoamericana puesto que, entre otros, el Programa de Acción de la VIII Cumbre de San Salvador de 2008 reiteró "el compromiso con la promoción y protección de los Derechos Humanos, a través del intercambio de experiencias y buenas prácticas en la materia, ampliando los espacios de cooperación y el apoyo al sistema internacional de la Organización de las Naciones Unidas", lo que no excluye la plena cooperación entre los sistemas regionales en los que participan los Estados de la región. El camino para esta colaboración está abierto. Así, además de algunos Seminarios y Jornadas que se ha celebrado, se podría destacar la reunión de casi "20 autoridades de la Corte Interamericana de Derechos Humanos, la Conferencia de Ministros de Justicia de los Países Iberoamericanos (COMJIB), la Asociación Iberoamericana de Ministerios Públicos (AIAMP), la Cumbre Judicial Iberoamericana y la Asociación Iberoamericana de Defensorías Públicas (AIDEF)", que tuvo lugar en julio de 2019 calificado como "Foro de Alto Nivel: Fortalecimiento del Estado de Derecho y los Derechos Humanos para lograr sociedades pacíficas, justas, inclusivas y transparentes"19.

La segunda consistiría en que los Tribunales regionales acogiesen el discurso y los contenidos expresados en las Conferencias y en los instrumentos iberoamericanos para fundamentar el reconocimiento y garantía de determinados derechos. Como decimos, esto es más posible específicamente en el caso de la Corte IDH. Así se explica, por ejemplo, que entre otras ${ }^{20}$ en la Sentencia de 27 de agosto de 2020, en el Caso Urrutia Laubreaux vs. Chile, la Corte hiciera una referencia explícita al Estatuto del Juez iberoamericano, aprobado por la VI Cumbre Iberoamericana de Presidentes de Cortes Supremas y Tribunales Supremos de Justicia, celebrada en Santa Cruz de Tenerife, Canarias, España, los días 23, 24 y 25 de mayo de 2001, y que, sin duda, forma parte de las instancias que delimitan los contornos del sistema iberoamericano. En este asunto se trató el reconocimiento de un derecho de carácter civil y político, en concreto, del derecho al debido proceso en el que el Estatuto del Juez Iberoamericano señala que "la responsabilidad disciplinaria de los jueces será competencia de los órganos del Poder Judicial legalmente establecidos, mediante procedimientos que garanticen el respeto del debido proceso y, en

perspectiva iberoamericana", 2009, en https:/www.segib.org/proteger-los-derechos-humanos-unreto-para-la-comunidad-iberoamericana/.

${ }^{19}$ Organización de las Naciones Unidas para la Educación, la Ciencia y la Cultura (UNESCO), Cuadernos de Discusión de Comunicación e Información, 15, 2019, p. 9.

${ }^{20}$ Vid., también; Corte IDH. Caso Villaseñor Velarde y otros Vs. Guatemala. Fondo, Reparaciones y Costas. Sentencia de 5 de febrero de 2019. Serie C No. 374, párr. 86.

Araucaria. Revista Iberoamericana de Filosofia, Politica, Humanidades y Relaciones Internacionales, año $23, \mathrm{n}^{\circ} 46$. Primer cuatrimestre de 2021. Pp. 631-652. ISSN 1575-6823 e-ISSN 2340-2199 https://dx.doi.org/10.12795/araucaria.2021.i46.31 
particular, el de los derechos de audiencia, defensa, contradicción y recursos legales que correspondan"21.

\section{Los derechos económicos, sociales y culturales: un espacio de aproximación entre el sistema iberoamericano y la Corte Interamericana de Derechos humanos}

La Conferencia iberoamericana no ha optado por servir de instancia en la que se promueva la elaboración y aprobación de acuerdos internacionales con valor vinculante por lo que no ha aprobado ningún instrumento específico y preciso sobre los derechos humanos y, en su caso, eventuales sistemas de garantía de estos derechos. Nada hace pensar que se haya suscitado, siquiera, esta posibilidad. Asimismo, sería engorroso determinar un listado o catálogo de derechos sobre la base de los postulados que se han expresado en las Cumbres iberoamericanas que, en la mayoría de los casos, ponen los énfasis en la realización de Programas y Políticas en campos de la cooperación iberoamericana. En definitiva, la Conferencia carece de un instrumento políticojurídico que detalle los derechos humanos en perspectiva iberoamericana porque un esfuerzo en esta dirección no produciría resultados tangibles. En realidad, los Estados iberoamericanos participan en Organizaciones internacionales, sobre todo, la Organización de Estados Americanos (OEA) y el Consejo de Europa que disponen de una relación detallada de los derechos fundamentales que se reconocen y que, además, establecen mecanismos de protección de estos derechos.

Esto no impide, sin embargo, que el sistema iberoamericano plasme su posición en relación con determinados derechos bien a través de los postulados de la Conferencia iberoamericana o de otras instancias del sistema. Este último sería el caso, por citar un ejemplo significativo, de la "Carta Iberoamericana de los Derechos y Deberes del Ciudadano en Relación con la Administración Pública", que fue aprobada por el Consejo Directivo del CLAD en 2013 y adoptada ese mismo año por la XXIII Cumbre Iberoamericana, celebrada en Ciudad de Panamá ${ }^{2}$. En todo caso, aclarar los logros que se han producido por iniciativa de las Cumbres y del sistema iberoamericano en el sector de los derechos humanos e identificar los sectores en los que la Corte IDH pudiera tener un papel más esencial exige valorar dos circunstancias de interés: Por una parte, la Conferencia iberoamericana y las instancias del sistema iberoamericano no han llevado a cabo una labor ordenada y sistemática en

\footnotetext{
${ }^{21}$ https://www.corteidh.or.cr/docs/casos/articulos/seriec 409 esp.pdf, p. 30, párr. 110.

22 Vid., en particular, Jaime Rodríguez-Arana Muñoz, J., "Sobre la Carta iberoamericana de los derechos y deberes del ciudadano en relación con la Administración Pública", Anuario da Facultade de Dereito da Universidade da Coruña, núm. 18, 2014, pp. 17-29.
} 
materia de derechos humanos que identifique los campos que deben ser objeto de especial preocupación para los Estados iberoamericanos; tampoco han regulado con precisión este campo de la cooperación iberoamericana describiendo los derechos que se reconocen y los eventuales sistemas de garantía; e, incluso, no se han detallado el contenido y alcance de los compromisos adquiridos por los Estados de la región. Pero, por otra parte, las declaraciones finales de la Conferencia y el quehacer de las instancias del sistema iberoamericano expresan postulados en el sector de los derechos humanos que permiten deducir que se ha querido poner el énfasis en los enfoques relativos a aquellos que tienen un contenido económico, social y cultural.

Sin olvidar el interés por la defensa de todos los derechos con independencia de su carácter, sería posible sostener que las Cumbres y las instancias del sistema iberoamericano inciden particularmente en los derechos de contenido social y cultural. Como se ha señalado, "la agenda de las cumbres experimentará un significativo giro social, centrando su atención especialmente en los derechos económicos, sociales y culturales"23. En la misma dirección se ha pronunciado Carlos F. Fernández Liesa al destacar que se ha expresado "una especial sensibilidad, en el espacio iberoamericano, por el desarrollo social" 24 . Esto deriva de dos razones: la primera, porque los ámbitos prioritarios y "naturales" de la cooperación iberoamericana tienen que ver precisamente con materias entreveradas de derechos sociales y culturales. Y la segunda porque las principales instancias del sistema iberoamericano ejercen su tarea sobre campos de contenido social y cultural como sucede con la Organización de Estados iberoamericanos (OEI), la Organización Iberoamericana de la Seguridad Social y la Organización Iberoamericana de la Juventud ${ }^{25}$.

Desde ahí, a la luz de las líneas jurisprudenciales de la Corte IDH, se desprende que los postulados de la Conferencia y del sistema iberoamericano podrían ser tenidos en cuenta por este órgano de protección de los derechos humanos, por cuanto inciden sobremanera en campos de contenido social y cultural. Desde luego, en el campo de los derechos económicos, sociales y culturales la Corte IDH ha abierto una vía de interés sobre todo a partir de la Sentencia de 31 de agosto de 2017, en el caso Lagos Del Campo vs. Perú ${ }^{6}$ en la que llega a afirmar que

${ }^{23}$ Celestino del Arenal, C., Democracia y derechos humanos, op. cit., p. 154.

${ }^{24}$ Carlos Fernández Liesa, La democracia y los derechos humanos, op. cit., p. 76.

${ }^{25}$ Una primera aproximación en Tomás Lozano Escribano, "La institucionalización de la Comunidad iberoamericana y algunas de sus posibles funciones en las actuales relaciones internacionales", Cuadernos de Estrategia, $\mathrm{n}^{\circ}$. 65, 1993, pp. 179-193; y El sistema iberoamericano la cooperación al servicio de la Comunidad, Madrid, Secretaría General Iberoamericana (SEGIB), 2016.

${ }^{26}$ De imprescindible lectura Eduardo Ferrer Mac Gregor; Mariela Morales Antoniazzi y Rogelio Flores Pantoja, Inclusión, Ius Commune y justiciabilidad de los DESCA en la jurisprudencia interamericana. El caso Lagos del Campo y los nuevos desafios, México, Instituto de Estudios Constitucionales del Estado de Qurétaro, 2018, en concreto los trabajos de Oscar Parra Vera, pp. 181234 y Jorge Calderán Gamboa, pp. 333-380.

Araucaria. Revista Iberoamericana de Filosofia, Politica, Humanidades y Relaciones Internacionales, año $23, \mathrm{n}^{\circ} 46$. Primer cuatrimestre de 2021. Pp. 631-652. ISSN 1575-6823 e-ISSN 2340-2199 https://dx.doi.org/10.12795/araucaria.2021.i46.31 
ha establecido previamente su competencia para conocer y resolver controversias relativas al artículo 26 de la Convención Americana, como parte integrante de los derechos enumerados en la misma, respecto de los cuales el artículo 1.1 confiere obligaciones generales de respeto y garantía a los Estados (...).

Siendo así que, además, "ha dispuesto importantes desarrollos jurisprudenciales en la materia, a la luz de diversos artículos convencionales". Por lo que:

en atención a estos precedentes, con esta Sentencia se desarrolla y concreta una condena específica por la violación del artículo 26 de la Convención Americana sobre Derechos Humanos, dispuesto en el Capítulo III, titulado Derechos Económicos, Sociales y Culturales de este tratado ${ }^{27}$.

Las posibilidades que crea esta sentencia permitirían acudir a instrumentos del sistema iberoamericano para fundamentar las posiciones de la Corte y reforzar la justiciabilidad directa de los derechos económicos, sociales y culturales. En cualquier caso, los aportes de la Conferencia y del sistema iberoamericano en esta materia servirán para la interpretación y precisión de los contenidos de los derechos que se reconozcan que, a partir de ahora, podrán gozar de una efectiva protección. Así es, como lo expresó en su voto razonado del juez Roberto F. Caldas, el asunto Lagos Del Campo vs. Perú

es una decisión histórica, que representa un gran paso jurisprudencial. Si bien, un demorado trayecto pero debidamente estudiado, reflexionado, ponderado y trabajado a lo largo de muchos años sobre la justiciabilidad de los Derechos Económicos, Sociales, Culturales y Ambientales (...) por diversas composiciones judiciales de este Tribunal de San José, y con ello la decisión de declarar violado por primera vez, en su historia jurisprudencial, el artículo 26 de la Convención Americana sobre Derechos Humanos ${ }^{28}$.

Por lo que en ulteriores asuntos la Corte IDH tiene a su cargo la tarea de precisar el contenido y alcance de los derechos económicos, sociales y culturales que se reconozcan y en esta labor sería de gran utilidad acoger los postulados normativos del sistema iberoamericano en materia de derechos humanos. Es evidente que

en el contexto latinoamericano, los argumentos tanto a favor como en contra de la justiciabilidad del artículo 26 de la CADH continúan en la agenda y se

\footnotetext{
${ }^{27}$ https://www.corteidh.or.cr/corteidh/docs/casos/articulos/seriec 340 esp.pdf, p. 51, párr. 154.

${ }_{28}$ Ibidem, p. 1, párr. 1. En la misma dirección el voto concurrente del juez Eduardo Ferrer MacGregor Poisot, p. 1, párr. 1. Sin embargo, también conviene la lectura de los votos parcialmente disidentes de los jueces Eduardo Vio Grossi, y Humberto Antonio Sierra Porto.
} 
enlazan con la necesidad de confluir una dogmática singular de los DESCA en el espacio jurídico interamericano ${ }^{29}$.

Esta dogmática se debe nutrir de las posiciones que los Estados expresan en instancias de distinto tipo y entre las que ocupan una posición privilegiada todas las piezas que integran el sistema iberoamericano $\mathrm{y}$, en especial, los resultados en el campo de los derechos económicos, sociales y culturales de la Conferencia iberoamericana.

\section{Dos sectores en los que la Corte Interamericana de Derechos humanos puede acoger postulados de las Cumbres y del sistema iberoamericano: migraciones y cultura}

El contexto político que rodea la Conferencia Iberoamericana no es idóneo ni favorable para construir un marco normativo e institucional en materia de derechos humanos, a pesar de que se destaque, con razón, la importancia de esta instancia de concertación y cooperación entre los Estados iberoamericanos ${ }^{30}$ e, incluso, sobresalga su dimensión política en la escena internacional ${ }^{31}$. La vigencia de esta instancia ha sido destacada por la actual Secretaria General Iberoamericana, Rebeca Grynspan, para quien "las Cumbres Iberoamericanas, ahora más que nunca, adquieren una importancia renovada, como un espacio que ha logrado evolucionar en el tiempo y ajustarse a distintas realidades" 32 , pero esto no garantiza que tenga la capacidad de cimentar una arquitectura político-jurídica en el sector de los derechos humanos con las notas de autonomía conceptual, normativa e institucional. Sin embargo, esta limitación no desmerece los logros que en los temas de derechos humanos se han producido en las Conferencia iberoamericanas. Por ello, una de las vías para asegurar la efectividad de los compromisos aceptados por los Estados iberoamericanos sería a través del quehacer de los tribunales de los sistemas regionales en los que participan los Estados de la región y, en especial, mediante las decisiones de la Corte IDH.

\footnotetext{
${ }^{29}$ Eduardo Ferrer Mac Gregor; Mariela Morales Antoniazzi y Rogelio Flores Pantoja, Inclusión, Ius Commune, op. cit., Presentación, p. 18.

${ }^{30}$ José Luis Rubio Cordón, "La Comunidad Iberoamericana: una causa”, Cuadernos de Estrategia, $n^{\circ} .92,1997$, pp. 349-377.

${ }^{31}$ Juan Pablo de Laiglesia "Las cumbres como mecanismo de cooperación política de la comunidad iberoamericana", Cuadernos de Estrategia, n. 126, 2004, pp. 73-98; y Susanne Gratius, "La Cumbre Iberoamericana de Salamanca: ¿de una comunidad cultural a una alianza política?, Iberoamericana. América Latina, España, Portugal: Ensayos sobre letras, historia y sociedad. Notas, Reseñas iberoamericanas, $\mathrm{n}^{\circ}$. 21, 2006, pp. 171-176.

${ }^{32}$ Rebeca Grynspan, "Las Cumbres Iberoamericanas, ahora más que nunca", Le Monde Diplomatique en español, $\mathrm{n}^{\circ} .274,2018$.
} 
El campo de los derechos humanos es un ámbito específico de la cooperación entre los Estados iberoamericanos. Esto ha sido aceptado en los instrumentos de la Conferencia iberoamericana, incluso con un sentido más amplio, como se hizo en la Declaración de Guadalajara, que atestigua el compromiso de los Estados de la región por conformar, con base en la cooperación, "un acervo iberoamericano en el ámbito de los derechos humanos que consolide conductas de respeto, libertad y armonía en lo político, lo jurídico, lo económico y lo social" ${ }^{33}$. Ahora bien, resulta complicado detectar ámbitos específicos en los que se hubiera construido una arquitectura normativa e institucional en esta materia. No obstante, conviene detenerse en dos instrumentos que abordan terrenos preferentes de la cooperación para los Estados iberoamericanos, curiosamente los dos aprobados en la Cumbre de Montevideo de 2006, porque los dos sectores son extremadamente propicios para que se produzcan pronunciamientos por parte de la Corte IDH.

En primer lugar, sobresale el Compromiso de Montevideo sobre Migraciones y Desarrollo, por el que culmina el discurso de la Cumbre en el sector de las migraciones pretendiendo dar respuestas a uno de los fenómenos que interesan singularmente a la Comunidad iberoamericana ${ }^{34}$. Esta comunidad, como se ha dicho, "sitúa el respeto de los derechos humanos en el centro a la hora de abordar el fenómeno de las migraciones" ${ }^{35}$. Desde el principio, este instrumento impone una óptica de las migraciones que se fundamente en la vigencia de los derechos humanos al proclamar "un análisis global" que se realice "desde una perspectiva integral y coherente (...) basado en el respeto a los derechos humanos"36. Desde ahí, se realizan indicaciones políticas y normativas que se podrían resumir así:

La primera y más trascendental es que las migraciones deben ser contempladas sobre la base de la persona del migrante. En palabras del Compromiso de Montevideo: "es imperativo situar la persona del migrante en el centro de los programas o proyectos migratorios, garantizando que las políticas migratorias respeten plenamente los derechos humanos de los migrantes $(\ldots)^{\prime 37}$.

\footnotetext{
${ }^{33}$ SEGIB: https://www.segib.org/wp-content/uploads/Primera-Cumbre-Iberoamericana-de-Jefesde-Estado-y-de-Gobierno.pdf.

${ }^{34}$ Una primera aproximación: Gonzalo Fanjul "Una agenda iberoamericana de las migraciones", Política Exterior, vol. 33, n 187, 2019, pp. 92-101; María de los Ángeles Cano Linares (Dir.), Migraciones Internacionales en el espacio iberoamericano del siglo XXI, Madrid, Dykinson, 2012; y Carmen Pérez González, "El codesarrollo como elemento de la cooperación en materia migratoria en el espacio iberoamericano", en Migraciones y desarrollo: II Jornadas Iberoamericanas de Estudios Internacionales, Montevideo, 25, 26 y 27 de octubre de 2006, Madrid, Marcial Pons, 2007, pp. 273-284.

${ }^{35}$ Cástor Miguel Díaz Barrado, "Migraciones y Comunidad Iberoamericana de Naciones. Un marco general", en Migraciones internacionales y co-desarrollo. El caso de Ecuador. Conclusiones y Propuesta del Seminario de Expertos sobre Co-desarrollo y derechos de las personas: El caso de Ecuador, 2011, Centro de Estudios de Iberoamérica, Cáceres, 2012, p. 20.

${ }^{36}$ SEGIB:https://www.segib.org/?document=compromiso-de-montevideo-sobre-migraciones-ydesarrollo.

${ }^{37}$ Ibidem.
}

Araucaria. Revista Iberoamericana de Filosofia, Política, Humanidades y Relaciones Internacionales, año $23, \mathrm{n}^{\circ} 46$. Primer cuatrimestre de 2021. Pp. 631-652. ISSN 1575-6823 e-ISSN 2340-2199 https://dx.doi.org/10.12795/araucaria.2021.i46.31 
Este postulado no es inocente puesto que no se prima la situación económica de los Estados, las consecuencias políticas de las migraciones en el espacio regional o la seguridad interna de los países, sino el respeto de los derechos de los migrantes. Por lo tanto, existe una obligación genérica de inundar las políticas, programas y acciones en materia de migración desde la perspectiva preferente de los derechos humanos. El instrumento aprobado en Montevideo avanza un poco más y precisa la obligación genérica imponiendo una pauta de conducta reflejada en que los Estados iberoamericanos deben "promover el fortalecimiento de los derechos humanos como un componente central de las políticas y prácticas migratorias", asegurando el respeto de los derechos humanos en los ordenamientos jurídicos internos ${ }^{38}$. En resumen, se proporciona un argumento político de calado para que los Estados de la región canalicen sus políticas en materia de migración por la ruta del respeto de los derechos humanos y no tanto por la custodia de otros intereses políticos o económicos.

La segunda indicación parapeta el fenómeno migratorio en el resguardo del sistema internacional de reconocimiento y protección de los derechos humanos, concebido en un sentido amplio, y abarcando todas las normas del ordenamiento jurídico internacional en la materia. El Compromiso de Montevideo lo expresa con nitidez al decir que

los Estados, al ejercer su derecho de regular el ingreso y la permanencia de personas en su territorio, deben respetar las normas del derecho internacional, de los derechos humanos, del derecho internacional humanitario y del derecho internacional de los refugiados, desde sus respectivos ámbitos jurídicos de aplicación ${ }^{39}$.

La aceptación de las normas internacionales en el campo de las migraciones es la condición para hacer efectiva la vigencia de los derechos humanos en el espacio iberoamericano y ello espolea a los Estados de la región para que se adhieran a los principios y normas que regulan los flujos migratorios en el plano internacional. Es decir, estas normas, una vez aceptadas por los países iberoamericanos, servirán de fundamento para su acogida en instrumentos específicamente iberoamericanos e interamericanos que regulen la migración.

Por lo tanto, uno de los sectores más seductores de las relaciones iberoamericanas ${ }^{40}$, el de las migraciones, abriga una concepción en la que se hace imprescindible el enfoque de los derechos humanos. Todo esto explica que

${ }^{38}$ Ibidem

${ }^{39}$ Ibidem

${ }^{40}$ Organización Internacional para las Migraciones. Estudio: Migración y Desarrollo en Iberoamérica, elaborado por el Observatorio Iberoamericano sobre Movilidad Humana, Migraciones y Desarrollo (OBIMID) e Instituto Universitario de Estudios sobre Migraciones (IUEM), Universidad Pontificia Comillas; Secretaría General Iberoamericana, s/f, disponible en https://www.segib. org/?document=estudio-migracion-y-desarrollo-en-iberoamerica-version-completa.

Araucaria. Revista Iberoamericana de Filosofia, Politica, Humanidades y Relaciones Internacionales, año $23, \mathrm{n}^{\circ} 46$. Primer cuatrimestre de 2021. Pp. 631-652. ISSN 1575-6823 e-ISSN 2340-2199 https://dx.doi.org/10.12795/araucaria.2021.i46.31 
la Corte IDH se haya hecho eco del contenido del instrumento iberoamericano. Así, en la Opinión Consultiva OC-21/14 de 19 de agosto de 2014 sobre Derechos y garantías de niñas y niños en el contexto de la migración y/o en necesidad de protección internacional, la Corte dice con toda rotundidad, recogiendo expresamente los términos del Compromiso de Montevideo, que los Estados se han comprometido a

promover el fortalecimiento de los derechos humanos como un componente central de las políticas y prácticas migratorias de los países de origen, de tránsito y de destino, asegurando la protección de los derechos humanos de los migrantes en el marco del ordenamiento jurídico de cada Estado, independientemente de su condición migratoria, y cualquiera que sea su nacionalidad, origen étnico, género o edad ${ }^{41}$.

En segundo lugar, la cultura es un ámbito especial de la cooperación iberoamericana, siendo así que está íntimamente vinculado y revela la identidad iberoamericana. Por lo menos,

las bases en las que se asienta la existencia de la Comunidad Iberoamericana y consiguientemente ese espacio cultural propio (...) hacen referencia (...) a una perspectiva o concepción del mundo, a una historia y un acervo cultural común y a unos principios y valores que conforman la identidad iberoamericana ${ }^{42}$.

Pero también la cooperación cultural iberoamericana puede ser enfocada desde la perspectiva de los derechos humanos, como se hace en uno de los más notables instrumentos político-jurídicos que han emanado de las Cumbres. La Carta Cultural Iberoamericana de $2006^{43}$, además de ser un texto que delinea los aspectos esenciales que deben estar presentes en la cooperación entre los países iberoamericanos en el plano cultural, adopta un enfoque en el que prima la vigencia de los derechos humanos. Así lo reconocían el entonces Secretario General Iberoamericano, Enrique V. Iglesias, y el Secretario General de la Organización de Estados Iberoamericanos para la Educación, la Ciencia y la Cultura (OEI), Álvaro Marchesi, al decir que la Carta

es un compromiso voluntario de cooperación, que surge de la solidaridad entre Estados y su visión integral de la cultura queda patente en la amplitud y variedad de los ámbitos de aplicación de la misma: los derechos humanos y los derechos culturales, las culturas tradicionales, indígenas, de afrodescendientes

\footnotetext{
${ }^{41}$ https://www.acnur.org/5b6ca2644.pdf, pp. 15-16, párr. 40.

${ }^{42}$ Celestino del Arenal, El acervo iberoamericano, op. cit., p. 10.

${ }^{43}$ Por todos, C. M. Díaz Barrado, "La carta cultural iberoamericana: una pieza básica para la configuración de un espacio cultural iberoamericano", en Derecho internacional y comunitario ante los retos de nuestro tiempo: homenaje a la profesora Victoria Abellán Honrubia, Madrid, 2009, pp. 131-146.
} 
y de poblaciones migrantes (...) o las relaciones de la cultura con otros ámbitos de las políticas públicas, como la educación, el ambiente $(\ldots)^{44}$.

En realidad, los derechos humanos se conciben como un ámbito particular de la cooperación cultural y, desde luego, el resto de los sectores indicados guarda una estrecha relación con el respeto de los derechos humanos, bien sea respecto a la protección de grupos vulnerables (en particular pueblos indígenas y comunidades de afrodescendientes) o bien en lo concerniente a derechos de contenido económico y social. El principio relativo a los derechos humanos impregna el contenido de la Carta Cultural y cimenta la cooperación cultural entre los países iberoamericanos. Lo que queda plasmado tajantemente en el Preámbulo de este instrumento al aseverar que

la cultura se debe ejercer y desarrollar en un marco de libertad y justicia, reconocimiento y protección de los derechos humanos, y de que el ejercicio y el disfrute de las manifestaciones y expresiones culturales, deben ser entendidos como derechos de carácter fundamental ${ }^{45}$.

Por lo tanto, el ámbito prioritario y esencial de la cooperación iberoamericana, como es la cultura, debe llevarse a cabo en el respeto de estos derechos tal y como son concebidos en los instrumentos internacionales.

La Carta Cultural iberoamericana extrae todas las consecuencias posibles de este enfoque tanto en lo que se refiere a los principios que deben regir la cooperación cultural entre los Estados de la región como en lo relativo a los ámbitos preferentes en los que los Estados deben llevar a cabo su cooperación cultural. El primero de los principios recogidos en la Carta señala que "los derechos culturales deben ser entendidos como derechos de carácter fundamental" y, en consecuencia, su ejercicio y pleno disfrute deben ser abordados dentro del "carácter integral de los derechos humanos" Asimismo, el primero de los ámbitos de actuación que traza la Carta Cultural es precisamente la relación entre "Cultura y Derechos Humanos", especificando

la importancia de reforzar el papel de la cultura en la promoción y consolidación de los derechos humanos" de tal modo que "el diseño y gestión de las políticas culturales se correspondan con la observancia, el pleno respeto y la vigencia de los derechos humanos ${ }^{47}$.

\footnotetext{
44 SEGIB, Carta Cultural Iberoamericana, XVI Cumbre Iberoamericana de Jefes de Estado y de Gobierno, Montevideo, Uruguay, 4 y 5 de noviembre de 2006 Presentación, en http:// culturasiberoamericanas.org/carta_cultural.php.

${ }^{45}$ Ibidem.

${ }^{46}$ Ibidem.

${ }^{47}$ Ibidem.
}

Araucaria. Revista Iberoamericana de Filosofia, Política, Humanidades y Relaciones Internacionales, año $23, \mathrm{n}^{\circ} 46$. Primer cuatrimestre de 2021. Pp. 631-652. ISSN 1575-6823 e-ISSN 2340-2199 https://dx.doi.org/10.12795/araucaria.2021.i46.31 
En consecuencia, la cooperación cultural iberoamericana, más allá de un enfoque puramente retórico, penetra en el sector de los derechos humanos como ámbito para hacer efectivos los derechos de contenido cultural.

Hasta donde alcanzo, la Corte IDH no ha acudido a referenciar este instrumento en las decisiones que ha adoptado siendo probable que esto se deba también a que no ha conocido de asuntos que llevarán necesariamente a considerarlo. Sin embargo, las específicas indicaciones de la Carta Cultural al contenido de los derechos de carácter cultural y a su relevancia en la protección de derechos de las culturas tradicionales, indígenas, de afrodescendientes y de poblaciones migrantes, le otorgan una posición relevante para definir la noción de "identidad cultural" que, como se sabe, ha sido objeto de tratamiento por la Corte IDH. Así, por ejemplo, entre otras muchas, en la Sentencia de 27 de junio de 2012 en el Caso Pueblo Indígena Kichwa de Sarayaku vs. Ecuador, la Corte desaprovechó una oportunidad inigualable para referirse a este instrumento iberoamericano, de tal modo que se limitó a decir que "dos instrumentos internacionales tienen particular relevancia en el reconocimiento del derecho a la identidad cultural de los pueblos indígenas: el Convenio $\mathrm{N}^{\circ} 169$ de la OIT sobre pueblos indígenas y tribales y la Declaración de las Naciones Unidas sobre los Derechos de los Pueblos Indígenas" y señaló que "varios instrumentos internacionales de UNESCO también desarrollan el contenido del derecho a la cultura y a la identidad cultural" ${ }^{\prime 4}$. Seguramente en la Carta Cultural Iberoamericana hubiera encontrado una base sólida para sus posiciones.

\section{Conclusiones}

La vigencia de los derechos humanos es uno de los rasgos más característicos de la Conferencia Iberoamericana y, al mismo tiempo, un sector de actuación del sistema iberoamericano. La composición del "acervo iberoamericano", en función de las decisiones de los países de la región, integra aspectos de contenido histórico y cultural, pero, también, valores y principios acogidos más recientemente por el Derecho Internacional, entre los que se encuentra el respeto de los derechos humanos. Sin embargo, el contexto político y económico, y también institucional, en que el que se desenvuelve la Conferencia iberoamericana hace difícil que se logren más resultados tangibles en materia de derechos humanos en la medida en que su acción queda condicionada por la pertenencia de los Estados iberoamericanos a otras instancias u Organizaciones internacionales que se ocupan intensamente de los

${ }^{48}$ CIDH: Cuadernillo de jurisprudencia de la Corte Interamericana de Derechos Humanos, $\mathrm{n}^{\mathrm{o}} 22$ : Derechos Económicos, Sociales, Culturales y Ambientales, 2019, p. 148, párr. 215. Disponible en https://www.corteidh.or.cr/cf/corteidh_movil/cuadernillos.cfm.

Araucaria. Revista Iberoamericana de Filosofí, Política, Humanidades y Relaciones Internacionales, año $23, \mathrm{n}^{\circ} 46$. Primer cuatrimestre de 2021. Pp. 631-652. ISSN 1575-6823 e-ISSN 2340-2199 https://dx.doi.org/10.12795/araucaria.2021.i46.31 
derechos humanos. La afirmación de la vigencia de los derechos humanos en el espacio iberoamericano no debe ser meramente retórica. Por el contrario, permite señalar orientaciones, recomendar conductas, indicar pautas de comportamiento e, incluso, promover la elaboración de instrumentos que adopten un enfoque en el que esté presente el respeto de los derechos humanos como línea fundamental de actuación. Pero, además, debe propiciar que los tribunales de los sistemas regionales de protección de los derechos humanos en los que participan los Estados de la región y, en especial la Corte IDH, recojan los instrumentos iberoamericanos para fundamentar sus posiciones en el reconocimiento de derechos.

Los amplios sectores de la cooperación entre los iberoamericanos están inundados de la perspectiva de los derechos humanos. En concreto, los campos relativos a la cooperación cultural y a la cooperación en materia de migraciones enseñan, a través de los instrumentos aprobados, que el espacio de diálogo, concertación y cooperación que representa la Conferencia iberoamericana únicamente se puede concebir sobre la base del respeto de los principios que inspiran la conformación de una comunidad, entre los que prima la vigencia de los derechos humanos. Las Cumbres iberoamericanas todavía están a tiempo de servir de punto de encuentro entre los dos principales sistemas de reconocimiento y protección de los derechos humanos $\mathrm{y}$, en particular, entre los órganos encargados de hacer efectivos los mecanismos de garantía. Y, en particular, el sistema interamericano ha iniciado el camino, sobre todo a través del reconocimiento por la Corte IDH de la justiciabilidad directa de los derechos económicos, sociales y culturales, de acoger los postulados e instrumentos en los que el sistema iberoamericano sustenta la vigencia de los derechos humanos. 


\section{Referencias Bibliográficas:}

Arenal Celestino del, y Nájera, Alfonso, La Comunidad Iberoamericana de Naciones: (pasado, presente y futuro de la politica iberoamericana de España), Madrid, CEDEAL, 1990.

Arenal Celestino del, El acervo iberoamericano, Valores, principios y objetivos de la comunidad iberoamericana, Secretaria General Iberoamericana, Madrid, 2006.

Arenal Celestino del, "Democracia y derechos humanos en las cumbres iberoamericanas. Teoría y práctica", en La Cumbre de Cádiz y las relaciones de España con América Latina, Madrid, Colección Escuela Diplomática, 19, 2013, pp. 147-163.

Cumbres Iberoamericanas 1991-2016. Recopilación de sus Declaraciones, Comunicados y Planes de Acción, (Tomo I, I-XIV y Tomo II, XV-XXV), Secretaría General Iberoamericana, Madrid, 2018.

Díaz Barrado, Cástor Miguel, Perfiles de la Comunidad Iberoamericana de Naciones (A la luz de las Cumbres Iberoamericanas, Tribuna Americana, Casa de América, Cáceres, 1994.

Fernández Liesa, Carlos, "La democracia y los derechos humanos en el espacio eurolatinoamericano e iberoamericano", en Perspectivas sobre las relaciones entre la Unión Europea y América Latina, Madrid, Universidad Carlos III/BOE, pp. 51-77.

Ferrer Mac Gregor, Eduardo; Morales Antoniazzi, Mariela; y Flores Pantoja, Rogelio, Inclusión, Ius Commune y justiciabilidad de los DESCA en la jurisprudencia interamericana. El caso Lagos del Campo y os nuevos desafíos, México, Instituto de Estudios Constitucionales del Estado de Qurétaro, 2018.

Gratius, Susanne, "La Cumbre Iberoamericana de Salamanca: ¿de una comunidad cultural a una alianza política?, Iberoamericana. América Latina, España, Portugal: Ensayos sobre letras, historia y sociedad. Notas, Reseñas iberoamericanas, $\mathrm{n}^{\circ}$. 21, 2006, pp. 171-176.

Grynspan, Rebeca, "Las Cumbres Iberoamericanas, ahora más que nunca", Le Monde diplomatique en español, $\mathrm{n}^{\circ}$. 274, 2018.

Laiglesia, Juan Pablo de, "Las cumbres como mecanismo de cooperación política de la comunidad iberoamericana", Cuadernos de Estrategia, $\mathrm{n}^{\circ}$. 126, 2004, pp. 73-98.

Lozano Escribano, Tomás, "La institucionalización de la Comunidad iberoamericana y algunas de sus posibles funciones en las actuales relaciones internacionales", Cuadernos de Estrategia, no. 65, 1993, pp. 179-193. 
Pérez González, Carmen, "El codesarrollo como elemento de la cooperación en materia migratoria en el espacio iberoamericano", en Migraciones y desarrollo: II Jornadas Iberoamericanas de Estudios Internacionales, Montevideo, 25, 26 y 27 de octubre de 2006, Madrid, Marcial Pons, 2007, pp. 273-284.

Pico de Coaña y de Valicourt, Yago, "El valor de los principios en la comunidad iberoamericana", Cuadernos de Estrategia, no. 126, 2004, pp. 149-203.

Rey Caro, Ernesto José, "Vigencia del Derecho Internacional en la Comunidad Iberoamericana de Naciones", en Foro de reflexión sobre el espacio jurídico iberoamericano: realizaciones y propuestas, Madrid, 2004, pp. 19-36.

Rubio Cordón, José Luis, "La Comunidad Iberoamericana: una causa", Cuadernos de Estrategia, $n^{\circ}$. 92, 1997, pp. 349-377. 